\title{
ANÁLISE DE CUSTOS ENTRE A RAQUIANESTESIA E A ANESTESIA VENOSA COM PROPOFOL ASSOCIADA AO BLOQUEIO PERIANAL LOCAL EM OPERAÇÕES ANORRETAIS
}

\section{Cost analysis between spinal and venous anesthesia with propofol associated with local perianal block in anorectal procedures}

\author{
Paulo Gustavo KOTZE, Cristiano Denoni FREITAS, Juliana Stradiotto STECKERT, Juliana Ferreira MARTINS, \\ Carlos Walter SOBRADO-JUNIOR, Luiz Carlos VON BAHTEN, Elisabeth Mila TAMBARA
}

ABCDDV/659

Kotze PG, Freitas CD, Steckert JS, Martins JF, Sobrado-Junior CW, Von Bahten LC, Tambara EM. Análise de custos entre a raquianestesia e a anestesia venosa com propofol associada ao bloqueio perianal local em operações anorretais. ABCD Arq Bras Cir Dig 2009;22(3):137-142

RESUMO - Racional - Atualmente cerca de $90 \%$ das operações anorretais podem ser realizadas em regime ambulatorial. A técnica anestésica é fator fundamental na busca de menor tempo de internamento e redução de custos nestes procedimentos. Não há consenso na literatura sobre qual o melhor tipo de anestesia para essas operações. Objetivo - Comparar os custos da técnica de raquianestesia com bupivacaína 0,5\% isobárica com a técnica de anestesia venosa com propofol associada ao bloqueio perianal local com lidocaína a $2 \%$ e bupivacaína $0,5 \%$ (anestesia combinada) em pacientes submetidos a operações anorretais. Métodos -Foram analisados dados de 99 pacientes submetidos à operações anorretais, divididos em dois grupos: grupo I (raquianestesia), composto por 50 pacientes e grupo II (anestesia combinada), composto por 49 pacientes. Foram estudados os procedimentos cirúrgicos, tempo de procedimento anestésico-cirúrgico, tempo de internamento e custos globais de cada paciente. Resultados Não houve diferença estatística significativa entre os grupos estudados em relação ao tipo de procedimento cirúrgico, sexo, idade e complicações. O tempo médio do procedimento anestésico-cirúrgico, no grupo I foi de 53,1 minutos e de 44,08 minutos no grupo II $(P=0,034)$. O tempo médio de internamento foi de 19,68 horas no grupo I e de 7,08 horas no grupo II $(\mathrm{P}<0,0001)$. O custo global médio no grupo I foi de $\mathrm{R} \$ 296,49$ e de

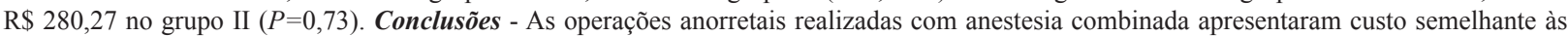
realizadas com raquianestesia. Houve menor tempo de procedimento anestésico-cirúrgico e menor tempo de internamento nos pacientes operados com anestesia combinada.

DESCRITORES: Anestesia. Cirurgia Colorretal. Canal Anal.

\section{INTRODUÇÃO}

As doenças orificiais, como exemplo, as hemorróidas, são muito comuns na população geral. Estima-se que cerca de $5 \%$ da população adulta nos Estados Unidos é afetada por tais doenças ${ }^{9}$. Elas podem ser tratadas de forma conservadora, todavia, o procedimento cirúrgico é necessário e imperativo em muitos casos.

A Sociedade Americana de Cirurgiões de Cólon e Reto, em seus protocolos práticos, autoriza a realização dos procedimentos cirúrgicos anorretais em regime ambulatorial em centros especializados com anestesia local, associada ou não à anestesia venosa ${ }^{10}$. Assim, na maioria dos grandes centros, o tratamento cirúrgico das doenças anorretais pode ser realizado de modo ambulatorial ${ }^{10}$. A anestesia para tais procedimentos é de grande importância.

Técnica anestésica ideal visa boas condições operatórias, agilidade no ambiente cirúrgico, ausência de efeitos colaterais, rápida recuperação com possibilidade de alta

Trabalho realizado no Serviço de Coloproctologia do Hospital Universitário Cajuru (SeCoHUC) - PUCPR, Curitiba, PR, Brasil.

Endereço para correspondência: Paulo Gustavo Kotze, e-mail: pgkotze@hotmail.com precoce e baixo custo 9 . Vários são os estudos publicados na literatura comparando diferentes técnicas anestésicas, demonstrando as suas vantagens e desvantagens ${ }^{9,10,17}$.

Quatro técnicas podem ser utilizadas: bloqueio regional espinhal (raquianestesia ou anestesia peridural), anestesia local, anestesia combinada (venosa + local) e anestesia geral. Nenhuma delas é considerada ideal, ou descrita como o padrão-ouro. A experiência do cirurgião, a posição do paciente na mesa cirúrgica, a experiência do anestesiologista e as condições do local são os fatores determinantes para a escolha de uma destas técnicas ${ }^{17}$.

A anestesia local pode ser realizada, com bons resultados. Entretanto, o desconforto para sua realização pode ser fator de dificuldade tanto para os pacientes como para os cirurgiões ${ }^{10}$. A combinação da anestesia venosa com propofol, permitindo inflitração perianal local sem dor ou reflexos, pode tornar os procedimentos mais toleráveis por parte dos pacientes, e com melhores condições transoperatórias para os cirurgiões?.

Em suma, ainda se busca uma técnica anestésica ideal para o tratamento cirúrgico das doenças anorretais, e estudos sobre o tema são constantemente publicados ${ }^{9,10,17}$.

Os objetivos deste estudo foram comparar os custos da técnica de raquianestesia com bupivacaína $0,5 \%$ isobárica 
com a técnica de anestesia venosa com propofol associada ao bloqueio perianal local com lidocaína a $2 \%$ e bupivacaína $0,5 \%$, o tempo do procedimento anestésico-cirúrgico e o tempo de internamento entre as duas técnicas.

\section{MÉTODO}

Este estudo foi realizado no setor de hospital-dia do Serviço de Coloproctologia do Hospital Universitário Cajuru (SeCoHUC - PUCPR), Curitiba, PR, Brasil. Em período de 18 meses, 106 pacientes foram submetidos à operações por doenças anorretais benignas e passaram a ser incluídos no banco de dados do Serviço de Coloproctologia. Dois tipos de anestesias para estas operações foram realizadas no período: raquianestesia e anestesia venosa com propofol associada ao bloqueio perianal local.

O projeto de pesquisa foi aprovado pelo Comitê de Ética em Pesquisa da PUCPR (CEP - PUCPR), sob o parecer número 241/07 e registro de número 1867 , permitindo a análise retrospectiva das informações destes pacientes.

Os critérios de inclusão foram: idade entre 16 e 80 anos, portadores de qualquer doença benigna anorretal com indicação cirúrgica; avaliação anestésica pré-operatória ASA I ou II; condição de compreensão do termo de consentimento informado; presença de acompanhantes e pacientes do sistema público de saúde.

Os critérios de exclusão foram: avaliação anestésica pré-operatória ASA III ou IV; concomitância de distúrbios mentais ou psiquiátricos documentados, que os impedissem de compreender e concordar com o termo de consentimento informado; ausência de acompanhantes; doenças anorretais com infecção aguda e pacientes portadores de planos de saúde e particulares.

Houve exclusão de sete pacientes, por não cumprirem os critérios. Dos 99 restantes, 50 foram operados com raquianestesia e 49 com anestesia combinada (propofol e bloqueio perianal local).

Todas as operações foram realizadas nas mesmas condições de ambiente. Os atos anestésicos foram sempre realizados pelos mesmos dois anestesiologistas do grupo de anestesiologia, e seguiram padronização técnica.

Os pacientes foram colocados em posição de decúbitodorsal, sobre a mesa cirúrgica e monitorizados com cardioscópio e oxímetro de pulso. Foi realizada punção venosa periférica, sempre no antebraço esquerdo, com cateter intravascular número 20. A partir deste ponto, iniciou-se a cronometragem do procedimento anestésico-cirúrgico. Passou-se então para a anestesia propriamente dita. Não se realizou antibioticoprofilaxia venosa nos pacientes incluídos no estudo.

Os pacientes do grupo I (raquianestesia) foram colocados na posição sentada, com ângulo de 90 graus entre a maca e sua coluna vertebral, em posição para facilitar a introdução da agulha no espaço intervertebral. Após antissepsia com álcool $70^{\circ}$ marcou-se o espaço L2/ L3 e anestesia local com lidocaína $1 \%$ sem vasoconstritor foi realizada na pele e planos adjacentes com $5 \mathrm{~mL}$ de solução. Posteriormente realizou-se a punção no referido espaço através da área da pele anestesiada até a penetração no espaço subaracnóide, com agulha especial para raquianestesia descartável número $27 \mathrm{G}$, com 88 x 0,42 mm. Após extravasamento de líquido cérebro-espinhal, injetou-se solução de $10 \mathrm{mg}$ de bupivacaína $0,5 \%$ isobárica, $2 \mathrm{~mL}$. Logo após, os pacientes foram colocados em posição de litotomia, com fixação dos membros inferiores nas perneiras cirúrgicas com ataduras de crepe. Realizou-se antissepsia da região anal com solução de iodopovidona tópica, na quantidade de $20 \mathrm{~mL}$ e colocação de campos estéreis, com preparo da mesa cirúrgica. Após o teste de sensibilidade, realizado com tração da pele perianal com pinça cirúrgica traumática, na ausência de dor por parte do paciente, iniciou-se o ato cirúrgico proctológico indicado para cada caso.

Os pacientes do grupo II (propofol venoso e bloqueio perianal local - anestesia combinada), após a monitorização e a punção venosa, foram sedados pelo anestesiologista com infusão de $2 \mathrm{mg} / \mathrm{kg}$ de propofol. Após a sedação inicial, os pacientes receberam quatro litros por minuto de oxigênio através de máscara facial. Posteriormente, foram colocados na posição de litotomia, com fixação dos membros inferiores de maneira idêntica a do grupo I. Procedeu-se à antissepsia da região anal com solução de iodopovidona tópica, na quantidade de $20 \mathrm{~mL}$ e posterior colocação dos campos estéreis. A solução anestésica local foi então preparada, com utilização de seringa de 20 $\mathrm{mL}$ e agulha de $40 \times 1,2 \mathrm{~mm}$ para aspiração de $10 \mathrm{~mL}$ de lidocaína a $1 \%$, sem vasoconstritor, associados a $10 \mathrm{~mL}$ de bupivacaína $0,5 \%$. O bloqueio anestésico perianal local foi realizado sempre pelo mesmo cirurgião, que foi responsável por todas as operações dos pacientes incluídos no estudo, com injeção da solução previamente descrita, com agulha $25 \times 0,6 \mathrm{~mm}$ pela técnica descrita por Schneider ${ }^{13}$. Esta técnica preconiza a anestesia da região perianal em leque, no subcutâneo, através de dois locais de punção, anterior mediano e posterior mediano, com injeção da solução anestésica após aspiração. A manutenção da anestesia foi realizada com infusão de $100 \mu \mathrm{g} / \mathrm{kg} /$ minuto de propofol até o final do ato operatório.

Houve controle na hidratação dos pacientes dos dois grupos durante os procedimentos, para prevenir-se retenção urinária. Restringiu-se para apenas $250 \mathrm{~mL}$ de soro fisiológico a $0,9 \%$ o volume injetado por paciente.

O término da cronometragem do tempo de procedimento anestésico cirúrgico foi marcado ao final da confecção do curativo perianal, nos dois grupos.

Após o término da operação anorretal, todos os pacientes foram mantidos em observação na sala de recuperação pós-anestésica imediata, com monitorização da oximetria de pulso. A alta do centro cirúrgico pela equipe de anestesiologia foi efetivada de maneira diferente nos dois grupos estudados.

Os pacientes do grupo I foram encaminhados ao leito de internação hospitalar, cerca de 60 minutos após o término da operação.Foram reavaliados no dia seguinte à operação e suas altas hospitalares am concluídas quando apresentavam condições.

Os pacientes do grupo II foram encaminhados a uma 
sala especial de repouso cerca de 60 minutos após o término da operação. Após a recuperação do seu nível de consciência, permaneceram nela até a alta ambulatorial definitiva pela equipe cirúrgica, na presença dos acompanhantes.

Todos os pacientes foram reavaliados após sete dias, em regime ambulatorial. Os prontuários foram avaliados dois meses após as operações, quando definitivamente finalizados pelo setor de faturamento, para que os seus custos globais fossem determinados que seguiu apenas o valor final total de cada caso operado faturado pelo hospital, não detalhado.

\section{Análise estatística}

As variáveis sexo, procedimentos cirúrgicos realizados (hemorroidectomias, fistulotomias $1 .{ }^{\circ}$ tempo, fistulotomias $2 .^{\circ}$ tempo, fissurectomias anais, ressecção de condilomas e de plicomas) e complicações (retenção urinária e cefaléia), por serem dicotômicas categóricas, foram analisadas inicialmente por teste de qui-quadrado. Para as variáveis idade e tempo de procedimento anestésico-cirúrgico, por serem as mesmas quantitativas contínuas, com pressuposto de normalidade atendido, foi aplicado teste $t$ de student. A análise do tempo de internamento e dos custos entre os grupos foi realizada pelo teste de Mann-Whitney, pela falta de normalidade entre as observações. O nível de significância (P) estabelecido foi de 5\%.

\section{RESULTADOS}

Foram operados 106 pacientes mas 99 pacientes foram incluídos no estudo. Dos sete excluídos, dois eram menores de 16 anos de idade e cinco apresentavam estado-físico pré-operatório ASA III.

A Tabela 1 ilustra os dados demográficos dos pacientes dos dois grupos. Não houve diferença estatística entre os mesmos em relação ao sexo e média de idade, o que demonstra a possibilidade de comparação.

TABELA 1 - Dados demográficos dos pacientes dos dois grupos estudados

\begin{tabular}{lccc}
\hline & I (raquianestesia) & $\begin{array}{c}\text { II (anestesia } \\
\text { combinada) }\end{array}$ & P \\
\hline $\mathrm{n}$ & 50 & 49 & \\
Sexo masculino & 22 & 24 & 0,619 \\
Sexo feminino & 28 & 25 & 0,619 \\
Média de idade (anos) & $44,2(17-71)$ & $39,31(17-80)$ & 0,075 \\
Desvio-padrão (anos) & 12,31 & 13,72 & \\
\hline
\end{tabular}

Com relação aos procedimentos cirúrgicos, no grupo I, foram realizadas 32 hemorroidectomias (associação com fissurectomia anal em cinco casos), nove fistulotomias em um tempo, seis fissurectomias anais com esfincterotomia interna, dois fistulotomias em dois tempos (segundo tempo - retirada do reparo esfincteriano e abertura do trajeto) e uma ressecção de plicomas anais de grandes dimensões (Figura 1).

No grupo II, foram realizadas 29 hemorroidectomias (associação com fissurectomia anal em três casos), nove

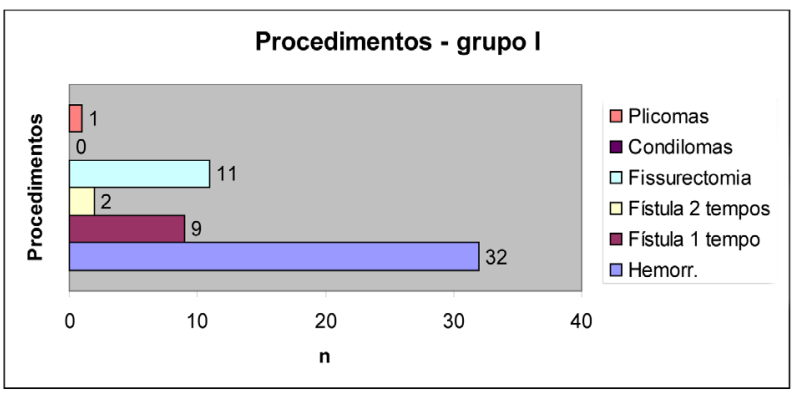

FIGURA 1 - Procedimentos cirúrgicos - grupo I

fistulotomias anais em 1 tempo, 4 fistulotomias em 2 tempos, 4 fissurectomias anais com esfincterotomia interna e 3 ressecções de condilomas anais. Não houve ressecção de plicomas anais neste grupo (Figura 2).

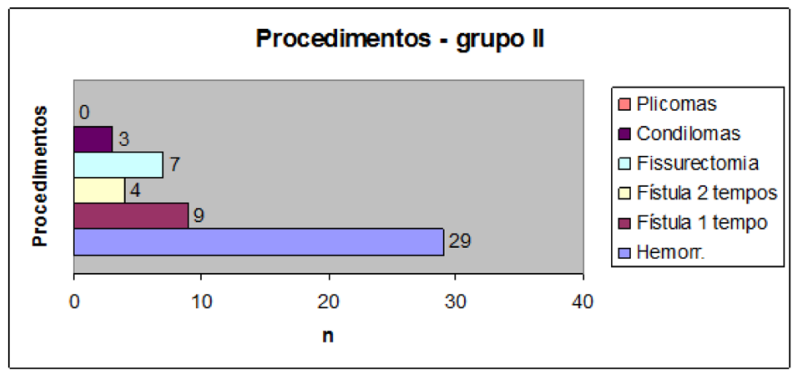

FIGURA 2 - Procedimentos cirúrgicos - grupo II

No grupo I foram realizados 55 procedimentos em 50 pacientes, por haver operações conjuntas em alguns casos. No grupo II, foram realizados 52 procedimentos em 49 pacientes. Para fins da análise estatística, foram considerados apenas o número de pacientes de cada grupo, e não o número de procedimentos (grupo I, $\mathrm{n}=50$; grupo II, $\mathrm{n}=49$ ).

Não houve diferença significante em nenhum dos procedimentos cirúrgicos realizados, analisados isoladamente entre os grupos. Portanto, permite-se afirmar que não houve diferença entre os grupos estudados na indicação cirúrgica.

O tempo do procedimento anestésico-cirúrgico, no grupo I, variou entre 20 e 120 minutos (média de 53,1 minutos e desvio-padrão de 22,2 minutos). No grupo II, houve variação entre 10 e 120 minutos (média de 44,08 minutos e desvio-padrão de 19,38 minutos). O nível de significância encontrado (P) foi de 0,034 . Houve diferença significante entre os dois grupos, e permite-se afirmar que os pacientes do grupo II tiveram menor tempo de procedimento quando comparados com o grupo I $\mathrm{I}^{7}$.

Em relação ao tempo de internamento, o grupo I apresentou valores entre 12 e 24 horas (média de 19,68 horas com desvio-padrão de 5,82 horas). No grupo II, houve variação entre 4 e 18 horas (média de 7,08 horas com desvio-padrão de 3,02 horas). Não houve valores discrepantes para serem excluídos na análise desta variável. O valor da significância $(\mathrm{P})$ encontrado foi de 5,71078E-20 $(\mathrm{P}<0,0001)$. Com este achado, permite-se afirmar que o tempo de internamento foi inferior no grupo II em comparação ao grupo I (Figura 3). 


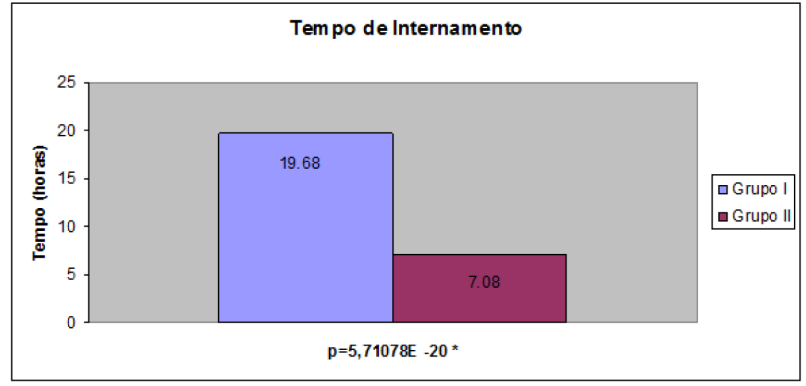

FIGURA 3 - Tempo de internamento nos grupos I e II (Método de Mann-Whitney)

O levantamento dos custos globais dos procedimentos realizados foi analisado. Nos pacientes do grupo I, ele variou entre $\mathrm{R} \$ 129,06$ e R \$ 1702,61 (média de R \$296,49 e desvio-padrão de R \$246,28). No grupo II variou entre R\$ 164,81 e R \$ 953,05 (média de R \$280,27 e desvio-padrão de R\$ 149,06). Para análise estatística, houve exclusão de sete pacientes do grupo I e de cinco do grupo II, MannWhitney com valores discrepantes. O nível de significância (P) encontrado foi de 0,73 , o que permite a conclusão de que não houve diferença de custos entre os dois grupos.

Sumário dos principais dados destas três variáveis, com média e valores máximo e mínimo, encontra-se detalhado na Tabela 2.

TABELA 2 - Sumário das principais variáveis, com desvio-padrão, valores mínimos e máximos

\begin{tabular}{lccccccccc}
\hline & \multicolumn{1}{c}{ Média } & \multicolumn{2}{c}{ Desvio-padrão } & \multicolumn{2}{c}{ Mínimo } & \multicolumn{2}{c}{ Máximo } & Valor dep \\
\hline Grupo & I & II & I & II & I & II & I & II & \\
\hline $\begin{array}{l}\text { Tempo Anestésico- } \\
\text { cirúrgico (minutos) }\end{array}$ & 53,10 & 44,08 & 22,20 & 19,38 & 20 & 10 & 120 & 120 & $0,034^{*}$ \\
$\begin{array}{l}\text { Tempo de interna- } \\
\text { mento (horas) }\end{array}$ & 19,68 & 7,08 & 5,82 & 3,02 & 12 & 4 & 24 & 18 & $<0,0001 *$ \\
$\begin{array}{l}\text { Custo global (reais) } \\
296,49\end{array}$ & 280,27 & 246,28 & 149,06 & 129,76 & 164,81 & 1702,61 & 953,05 & 0,73 \\
\hline
\end{tabular}

\section{DISCUSSÃO}

Cerca de $5 \%$ da população adulta americana é portadora de doenças anorretais6. Em torno de $10 \%$ destes pacientes apresentam necessidade de operações para adequado tratamento ${ }^{1}$.

A escolha da anestesia para operações sobre o reto e o ânus é um dos fatores fundamentais para o sucesso das mesmas. Não há consenso na literatura sobre qual a melhor técnica anestésica para este fim $^{9,10}$. Poucos trabalhos são conclusivos em determinar qual delas é a melhor. Fleischer et al., em 1994, demonstraram que a anestesia local é superior à anestesia epidural em operações proctológicas, por menores complicações e custos² ${ }^{2}$.

O domínio de todas diferentes técnicas anestésicas e a escolha da melhor opção para cada caso significa o bom senso por parte da equipe médica assistente para se alcançar o melhor para o paciente. Entretanto, em meio médico altamente influenciado pelas operadoras e planos de saúde, as políticas são agressivas em relação à economia. Os custos com os procedimentos atualmente constituem preocupação primordial na maioria dos países.
A curta permanência no hospital está diretamente ligada a menores custos para o tratamento cirúrgico de doenças anorretais $^{14}$. O que determina a possibilidade da realização de procedimentos cirúrgicos proctológicos em regime ambulatorial, entre outros fatores, é a escolha de técnica anestésica que permita a posterior recuperação pós-operatória dos pacientes em seus domicílios, sem complicações ou efeitos colaterais. Atualmente, cerca de $90 \%$ dos pacientes portadores de doenças anorretais podem ser operados de forma ambulatorial ${ }^{9}$. Escolher uma técnica anestésica que possibilite esta condição, com baixos custos e com boa eficácia, com conforto para os pacientes e equipe cirúrgica, é objeto de alguns estudos na literatura mundial ${ }^{9,10,17}$. Há serviços que utilizam como primeira opção a anestesia venosa associada ao bloqueio local ${ }^{11}$. Outros descrevem que procedimentos ambulatoriais podem também ser realizados com raquianestesia posterior ${ }^{5}$. A ampla discussão sobre qual a mais adequada técnica de anestesia para operações proctológicas motivou a realização desta análise.

Além da busca por redução de custos, outro fator foi a escassez de leitos de internação e lotação nos hospitais públicos no nosso meio. No Hospital Universitário Cajuru, há falta de vagas tanto nos centros cirúrgicos quanto nas enfermarias. Nos últimos anos, houve constante suspensão de procedimentos eletivos por falta de vagas. Devido à intensa movimentação dos ambulatórios, há grande acúmulo de casos em lista de espera para operações proctológicas. A literatura nacional apresenta alguns estudos citando esta realidade de alguns hospitais universitários brasileiros, mostrando as amplas vantagens possibilitadas com a realização destas operações em regime ambulatorial ${ }^{12,15}$.

A população estudada foi submetida à operações que seguem os padrões técnicos consagrados. Os dois grupos apresentaram homogeneidade nas suas características, de acordo com a análise estatística realizada, pois não houve diferença significativa entre os grupos nas variáveis sexo, idade e procedimento cirúrgico realizado. A diferença entre os dois grupos estudados foi apenas na técnica anestésica empregada, amplamente citadas na literatura como seguras e efetivas ${ }^{10}$.

Todos os pacientes incluídos no estudo foram do sistema público de saúde. Não foram analisados pacientes com planos de saúde e particulares. Os custos de procedimentos nestes pacientes seriam maiores. As evidentes diferenças nos valores da remuneração dos profissionais e da instituição justificaram esta decisão.

Boa parte dos estudos comparando técnicas anestésicas diferentes para operações anorretais foca sua população em pacientes submetidos a hemorroidectomias ${ }^{3,8}$. Este estudo incluiu igualmente pacientes portadores de outras doenças anorretais benignas com indicações cirúrgicas, como fissuras anais, fístulas e condilomas. Há mínima possibilidade de viés neste aspecto, visto que o porte cirúrgico, os tempos operatórios e os materiais utilizados nestas operações são equivalentes ${ }^{16}$. Infecções agudas perianais foram excluídas por se tratarem de procedimentos de emergência, não realizados de acordo com os requisitos técnicos deste estudo.

Assim como a técnica anestésica, a técnica de bloqueio 
perianal local no grupo II e a técnica cirúrgica utilizada nos pacientes seguiram minuciosa sistemática. Todos os pacientes foram operados pelo mesmo cirurgião. Todos os bloqueios perianais locais nos pacientes do grupo II foram realizados igualmente pelo mesmo cirurgião com a mesma técnica ${ }^{13}$.

Os achados comparativos entre os grupos relacionados ao tempo de procedimento anestésico-cirúrgico (medição indireta de tempo de ocupação da sala cirúrgica) mostraram diferença significativa. Houve redução do tempo de realização destes procedimentos nos pacientes do grupo II (tempo médio de 44,08 minutos), quando comparados ao grupo I (53,1 minutos), com $P=0,0339$. Pode-se atribuir este fato a múltiplos motivos ${ }^{7}$. Primeiramente, há necessidade de antissepsia das mãos do anestesiologista para a realização das raquianestesias. Em segundo lugar, a técnica de raquianestesia pode ser demorada por dificuldades técnicas em determinados pacientes. Além disso, uma espera significativa de tempo para que o efeito do anestésico seja efetivado muitas vezes se faz necessária. Todos estes fatores, entre outros, podem justificar maior tempo de realização de uma raquianestesia, visto que aplicação venosa de propofol claramente é mais rápida ${ }^{8}$. Com estes achados, pode-se afirmar que o tempo de ocupação da sala cirúrgica é menor com a anestesia combinada ${ }^{7}$ Resultados semelhantes foram encontrados por importantes estudos da literatura ${ }^{3,7,17}$.

No presente estudo, o tempo de internamento se mostrou reduzido nos pacientes submetidos à anestesia combinada (grupo II), quando comparados aos com raquianestesia (grupo I). Tais achados foram compatíveis com os estudos da literatura que comparam diferentes técnicas ${ }^{9,17,11,103,8,12}$.

A literatura brasileira apresenta muitos trabalhos interessantes demonstrando os benefícios das operações anorretais ambulatoriais $\mathrm{s}^{4,7,8,12,15}$. Entretanto, apenas dois estudos salientam a redução dos custos relacionados a estes procedimentos $^{8,15}$.

Os achados do presente estudo são compatíveis com os da literatura nacional e internacional, demonstrando baixos custos em operações ambulatoriais associadas a anestesia combinada (venosa e local). O custo médio calculado foi baseado nos custos globais do procedimento, ou seja, no total recebido pela instituição referente a estes procedimentos, da entrada do paciente até a alta hospitalar. $\mathrm{Na}$ análise dos custos dos procedimentos incluídos no presente estudo, houve alguns valores discrepantes da maioria dos valores achados. Enquanto a média de custos foi de R \$296,49 no grupo I e de $\mathrm{R} \$ 280,27$ no grupo II, alguns procedimentos tiveram custos mais altos, muito acima da média encontrada (custo máximo de $\mathrm{R} \$ 1702,61$ no grupo I e R \$ 953,05 no grupo II). Tal fato pode ser justificado pela utilização de outras medicações e materiais por parte dos pacientes, de uso contínuo, não-ligadas aos procedimentos anorretais. O detalhamento das contas hospitalares não foi realizado neste estudo. Entretanto, para fins de análise estatística dos custos, houve a escolha do método de Mann-Whitney, pela capacidade deste em excluir valores discrepantes sem prejudicar a análise final da variável.

Uma economia de apenas R $\$ 16,22$ (ou US\$ 8,11) para cada paciente pode ser projetada segundo os dados do presente trabalho. Para a casuística de 50 pacientes, uma estimativa de economia de R $\$ 811,00$ (ou US\$ 405,50 ) pôde ser referida em todo o estudo. A diferença de custos entre os dois grupos foi pequena e não apresentou significância estatística. Este dado não corrobora os achados da literatura, que mostram evidente diminuição de custos nos procedimentos ambulatoriais. Uma provável justificativa é a utilização do propofol no grupo II, medicação considerada de maior custo se comparada a outros anestésicos utilizados para o mesmo propósito. A não utilização de outros sedativos nos pacientes deste grupo aumenta a quantidade de propofol necessária para manter o paciente adequadamente sedado, o que diretamente aumenta os custos dos procedimentos. Além disso, a maioria dos estudos de análise de custos para procedimentos ambulatoriais foi com anestesia local isolada, o que tornou o método menos oneroso. Por outro lado, o conforto dos pacientes é menor por estarem acordados durante o bloqueio perianal local ${ }^{9,10,17}$.

\section{CONCLUSÕES}

As operações anorretais realizadas com anestesia combinada (propofol venoso associado ao bloqueio perianal local) apresentaram custo semelhante às realizadas com raquianestesia. Houve menor tempo do procedimento anestésico-cirúrgico e menor tempo de internamento nos pacientes operados com anestesia combinada. 
Kotze PG, Freitas CD, Steckert JS, Martins JF, Sobrado-Junior CW, Von Bahten LC, Tambara EM. Cost analysis between spinal and venous anesthesia with propofol associated with local perianal block in anorectal procedures. ABCD Arq Bras Cir Dig 2009;22(3):137-142

ABSTRACT - Background - Approximately ninety percent of anorectal surgical procedures are performed in ambulatory basis. The choice of a proper anesthetic technique is important to achieve shorter hospital stay and low costs. There's no evidence in the literature that an ideal type of anesthesia for these procedures exists. Aim - To compare the costs of patients operated with spinal anesthesia ( $0,5 \%$ bupivacaine) with combined anesthesia (propofol and local perineal block with 2\% lidocaine and 0,5\% bupivacaine) in anorectal surgical procedures. Methods - Data from 99 patients submitted to anorectal operations were retrospectively analyzed. They were divided in two groups: 50 patients were operated with spinal anesthesia (group I) and 49 with combined anesthesia (group II). The type of operation, the time of hospital stay, the time of anesthetic and surgical procedure and the costs were analyzed. Results - There were no significant differences between the studied groups regarding the type of operation, gender, age and complications. The time of anesthetic and surgical procedures was 53,1 minutes in group I and 44,08 minutes in group II $(P=0,034)$. The average time of hospital stay was 19,68 hours in group I and 7,08 hours in group II ( $\mathrm{P}<0,0001)$. The average cost of the procedures was R $\$ 296,49$ in group I and R $\$ 280,27$ in group II $(P=0,73)$. Conclusions - Anorectal procedures with combined anesthesia (propofol and local perineal block) had similar costs than those with spinal anesthesia. There was a lower time of anesthetic and surgical procedures and shorter hospital stay in the combined anesthesia group.

HEADINGS - Anesthesia. Colorectal surgery. Anal canal.

\section{REFERÊNCIAS}

1. Bleday R, Pena JP, Rothemberger DA, Golberg SM, Buls JG. Symptomatic hemorrhoids: current incidence and complications of operative therapy. Dis Colon Rectum 1992; 35(5):477-82.

2. Fleischer M, Marini CP, Statman R, Capella J, Shevde K . Local anesthesia is superior to spinal anesthesia for anorectal surgical procedures. Am Surg 1994; 60(11):812-5

3. Haveran LA, Sturrock PR, Sun MY. Simple harmonic scalpel hemorrhoidectomy utilizing local anesthesia combined with intravenous sedation: a safe and rapid alternative to conventional hemorrhoidectomy. Int J Colorectal Dis 2007; 22(7):801-6.

4. Henriques AC, Horta SHC, Pezzolo S, Waisberg J, Boratto SDF, Helal S, Gomes M, Speranzini MB. Procedimentos cirúrgicos em pacientes proctológicos selecionados sob anestesia local: estudo de 150 casos. Arq Gastroenterol 2000; 37(3):158-61.

5. Imbelloni LE, Vieira EM, Gouveia MA, Cordeiro JA. Raquianestesia posterior para cirurgias anorretais em regime ambulatorial: estudo piloto. Rev Bras Anestesiol. 2004; 54(6):774-80.

6. Johanson JF, Sonnenberg A. The prevalence of hemorrhoids and chronic constipation: an epidemiologic study. Gastroenterology 1990; 98(2):380-6.

7. Kotze PG, Tambara EM, Von Bahten LC, Silveira F, Wietzikoski E. Influência da técnica de anestesia no tempo de ocupação de sala cirúrgica nas operações anorretais. Rev bras Coloproct 2008; 28(2):227-233.

8. Lacerda Filho A, Melo JRC. Hemorroidectomia em regime ambulatorial sob anestesia local: estudo prospectivo de 50 casos. Rev bras Coloproct 1995; 15(4):206-10.
9. Li S, Coloma M, White PF, Watcha MF, Chiu JW, Li H. Comparison of the costs and recovery profiles of three anesthetic techniques for ambulatory anorectal surgery. Anesthesiol 2000; 93(5):1225-30.

10. Place R, Hyman N, Simmang C, Cataldo P, Church J, Cohen J. Practice parameters for ambulatory anorectal surgery. Dis Colon Rectum 2003; 46(5):573-6.

11. Read TE, Henry SE, Hovis RM, Fleshman JW, Birnbaum EH, Caushaj PF, Kodner IJ. Prospective evaluation of anesthetic technique for anorectal surgery. Dis Colon Rectum 2002; 45(11):1553-60.

12. Saad-Hossne R, Prado RG, Bakony Neto A. Cirurgia ambulatorial em proctologia: análise retrospectiva de 437 casos. Arq Gastroenterol 2005; 42(3):136-8.

13. Schneider H.C. Hyaluronidase with local anesthesia in anorectal surgery. Am J Surg. 1954;88(5): 703-6.

14. Sobrado CW. Cirurgia ambulatorial em proctologia: passado, presente e futuro. Arq Gastroenterol 2005; 42(3):133-5.

15. Sobrado CW, Nahas SC, Marques CF, Habr-Gama A. Cirurgia ambulatorial sob anestesia local em proctologia: experiência e análise do resultado de 503 operações. Rev bras Coloproct 2001; 21(4):228-33.

16. Smith LE. Ambulatory surgery for anorectal diseases: an update. South Med J 1986; 79(2):163-6.

17. Sun MY, Canete JJ, Friel JC, McDade J, Singla S, Paterson CA, Counihan TC. Combination propofol/ ketamine is a safe and efficient anesthetic approach to anorectal surgery. Dis Colon Rectum 2006; 49(7):1059-65.

Fonte de financiamento: não há Conflito de interesse: não há Recebido para publicação: 12/03/2009 Aceito para publicação: 28/05/2009 